\title{
Perspective
}

\section{Patient Safety and Healthcare Quality: The Case for Language Access}

\section{Cheri C. Wilson*}

Hopkins Center for Health Disparities Solutions, Department of Health Policy and Management, Johns Hopkins Bloomberg School of Public Health, The Johns Hopkins University, USA

Received: 9 November 2013, Accepted: 26 November 2013, ePublished: 27 November 2013

\section{Abstract}

This paper aims to provide a description of the need for Culturally and Linguistically Appropriate Services (CLAS) for Limited English Proficient (LEP) patients, an identification of how the lack of CLAS for LEP patients can compromise patient safety and healthcare quality, and discuss barriers to the provision of CLAS.

\section{Keywords}

Culturally and Linguistically Appropriate Services (CLAS), Limited English Proficient (LEP), Patient Safety, Healthcare Quality, Language Access

\section{Introduction}

\section{Could this have happened at your hospital?}

The following scenario, which takes place in a fictional U.S. hospital emergency room, was adapted from the DVD, Breaking down the Language Barrier: Translating Limited English Proficiency into Practice produced by the U.S. Department of Justice Civil Rights Division (1). Although fictional, these types of situations occur every day in hospitals worldwide.

A non-English speaking Vietnamese patient presents to the Emergency Department clutching his arm. The Patient Services Coordinator (PSC) cannot identify the language the patient speaks. She proceeds to speak loudly and slowly in English and asks the patient to sign the consent form for treatment. A nurse appears and asks the problem. The PSC states that the patient does not speak English. The nurse remembers that a computer technician speaks an Asian language. The nurse calls her and asks if she can interpret for the patient. The patient states his problem, but the computer technician, who speaks Thai, cannot understand him. She is able to determine that he speaks Vietnamese. The PSC and nurse recall that one of the receptionists speaks Vietnamese, but she has already left for the day. Neither the PSC nor nurse makes any further attempts to contact a qualified Vietnamese interpreter. The nurse brings the patient to the treatment room to be seen by the physician. The physician tries to conduct a history and physical examination through speaking English loudly and slowly as well as with hand gestures, while the patient continues to clutch his arm. The physician orders the nurse to give the patient codeine for the pain. The patient takes the medication. When the nurse returns to the room, the patient is found down on the floor. The nurse calls a code and it appears that the patient has had a severe adverse drug reaction to the Codeine (1).

How could this patient encounter have been improved? What could this hospital have done to ensure this patient's safety, e.g avoid an adverse drug reaction, and improve healthcare quality? A public service announcement produced by the Texas Association of Healthcare Interpreters and Translators (TAHIT) serves a similar purpose (2).

Scope of the problem: changing U.S. and state demographics As the U.S. becomes increasingly diverse, healthcare organizations struggle to provide CLAS for LEP patients. As of 2010, almost 40 million (12.7\%) of U.S. residents were foreign born, 57 million (20.6\%) spoke a language other than English at home, and 25 million (8.7\%) spoke English less than "very well" and were considered LEP (3,4). Between 1990 and 2010, the U.S. LEP population increased by $80 \%$. Between 1990 and 2010, the 10 states experiencing the greatest growth in their LEP populations were: Nevada (398.2\%), North Carolina (395.2\%), Georgia (378.8\%), Arkansas (311.5\%), Tennessee (281.4\%), Nebraska (242.2\%), South Carolina (237.2\%), Utah (235.2\%), Washington (209.7\%), and Alabama (202.1\%). In 8 states, at least $10 \%$ of the overall population is already LEP--California (19.8\%), Texas (14.4\%), New York (13.5\%), New Jersey (12.5\%), Nevada (12.3\%), Florida (11.9\%), Hawaii (11.8\%), and Arizona (9.9\%) (5). In addition, over 300 languages are spoken in the United States.

\section{Federal legislation and mandates}

In order to manage these issues, a commitment is needed at the national level, often in the form of legislation and mandates. For example, in the U.S., Title VI of the Civil Rights Act of 1964 considers the denial or delay of medical care due to language barriers to be discrimination based upon national origin. Any medical facility receiving federal funds (Medicaid or Medicare), must provide language assistance to LEP patients (6). Similarly, in 2001, the U.S. Department of Health and Human Services, Office of Minority Health issued fourteen national standards for culturally and linguistically appropriate services for healthcare

${ }^{\star}$ Corresponding author: Cheri C. Wilson; Email: chwilson@jhsph.edu 
organizations. These standards were organized into three themes--Culturally Competent Care (Standards 1-3), Language Access Services (Standards 4-7), and Organizational Supports for Cultural Competence (Standards 8-14) (7). The CLAS standards were designed to ensure that all people entering the healthcare system received equitable and effective treatment in a culturally and linguistically appropriate manner. The CLAS standards underwent an enhancement process during 2011 and 2012 and the final standards were released on April 24, 2013. The standards now include a principal standard in which the rest are organized into three themes-governance, leadership, and workforce, communication and language assistance, and engagement, continuous improvement, and accountability. Dr. Thomas LaVeist, the Director of the Hopkins Center for Health Disparities Solutions, Johns Hopkins Bloomberg School of Public Health, was a member of the National Project Advisory Committee for the CLAS Standards Enhancement Initiative (8).

\section{Patient safety and healthcare quality}

In Unequal Treatment, the importance of linguistic concordance to patient-provider communication was emphasized as a means to create an accurate medical and social history and assessment of the patient's cultural health beliefs (9). Without this concordance, the ability to communicate is compromised. According to The Joint Commission, from 1995 to 2002, communication was the root cause of almost $65 \%$ of sentinel events, unexpected events, which caused death or serious physical or psychological injury (10). Adverse consequences associated with ineffective communication can affect all patients and can lead to misunderstandings of a patient's concerns, misdiagnosis, unnecessary testing, poor patient compliance, inappropriate follow up, and poor patient satisfaction (9).

Research studies have documented that the safety and healthcare quality of LEP patients can be diminished due to language barriers (11). One study found that in $46 \%$ of emergency department cases, no interpreter was used for LEP patients. In addition, only $23 \%$ of teaching hospitals train physicians how to work with an interpreter $(11,12)$. A study analyzed 1,083 adverse incident reports from 6 Joint Commission-accredited hospitals for LEP vs. English-speaking patients for 7 months in 2005. This study found that a greater percentage of LEP patients experienced physical harm vs. English-speaking patients, $49.1 \%$ and $29.5 \%$ respectively. The LEP patients also experienced higher levels of physical harm ranging from moderate temporary harm to death, $46.8 \%$ and $24.4 \%$ respectively (13).

In an effort to provide language services, healthcare providers have sometimes resorted to drastic measures. At one hospital, the emergency room used the Yellow Pages to find a restaurant that spoke a particular language and would ask one of the restaurant employees to interpret over the phone (14). Other hospitals have used untrained support staff, strangers found in the waiting room or on the street, taxi cab drivers, etc $(11,12)$. In many instances, a family member serves as an interpreter, which raises privacy and other concerns. For example, California State Senator Leland Yee remembers translating for his mother at the doctor's office when he was only six years old. Several states have introduced legislation forbidding children under sixteen from serving as interpreters $(11,12,14)$. Untrained interpreters are more likely to commit errors in interpretation that can lead to adverse clinical consequences (12). Concerns about untrained interpreters include: lack of knowledge of medical terminology and confidentiality, their priorities may conflict with those of the patients, and their presence may inhibit discussions of sensitive issues, such as: domestic violence, substance abuse, psychiatric illness, and sexually transmitted diseases (11). Even at healthcare organizations with ample CLAS resources, providers chose to "get by" without an interpreter by: communicating through gestures, using limited second language skills, relying on histories obtained by other physicians, using patients' family members as ad hoc interpreters (15). Ebden et al. documented the perils of using ad hoc interpreters. They found that $23 \%$ to $52 \%$ of words and phrases were incorrectly interpreted in a study that recorded and analyzed ad hoc interpretation encounters (16).

Occasionally, a bilingual healthcare provider may be present. However, this is not without its problems as well. In one case, a mother whose daughter had fallen off her tricycle, lost custody for 48 hours because the doctor misinterpreted two Spanish words (Se pegó) as "I hit her" instead of "She hit herself" (17). Care can be compromised or delayed in the absence of any language services (trained or untrained). In other instances, the consequences can be catastrophic. For example, a healthcare team misunderstood an eighteen-year-old man who said that he was intoxicado. The team misunderstood the term to mean "intoxicated" rather than "nauseated." As a result, the patient was treated for a drug overdose for thirty-six hours before the doctors realized that he had a brain aneurysm. He ended up being a quadriplegic and his family was awarded $\$ 71$ million in a malpractice settlement (18).

\section{Barriers to providing CLAS}

Healthcare organizations and providers cite several barriers to providing CLAS, such as staff have no means of identifying patients who need CLAS before they arrive at the hospital; cost/reimbursement concerns, lack of tools and training resources, lack of community-level data, and staff generally feel uncomfortable asking patients to provide information about their primary language (19). If a healthcare organization has a robust mechanism for the collection of Race, Ethnicity, and Language (REAL) data, identifying patients needing CLAS should not be an issue. U.S. Census data can also help a healthcare organization identify its local patient population and the needs associated with that population.

Concerning cost/reimbursement, despite federal laws and current and proposed accreditation standards for CLAS, healthcare organizations and providers bear the burden of the cost of providing CLAS. An American Medical Association (AMA) survey found that the cost of interpreter services ranged from $\$ 30$ to $\$ 400$ per hour, while the average Medicaid office visit reimbursement was only $\$ 30$ to $\$ 50$. Private payers generally do not reimburse for interpreter services. In one study, only $3 \%$ of hospitals received direct reimbursement for language services. Medicaid (78\%) was the largest source, while private payers $(2 \%)$ were the smallest source. Although under fee-for-service Medicaid and State Children's Health Insurance Program (SCHIP), the use of medical interpreters is a reimbursable expense, only thirteen states do. In 2002, the Office of Management and Budget estimated that the cost of requiring payers to cover the cost of interpreters would only be about $\$ 4$ per patient for ED, inpatient, outpatient, and dental visits $(19,20)$. 


\section{Conclusion}

In the revised scenario on the Breaking down the Language Barrier: Translating Limited English Proficiency into Practice DVD, the following occurred.

When the patient arrived, the PSC pulled out a language identification card and asked the patient to point to the language he spoke. The patient pointed to Vietnamese. This time the PSC did not have to rely upon an ad hoc interpreter because a language assistance plan was in place. The PSC called a number and a qualified medical interpreter who spoke Vietnamese appeared. The interpreter, Ms. Nguyen, introduced herself to the patient and explained her role. She asked the patient his name, which was Mr. Than Pham. Speaking through the interpreter, the PSC asked Mr. Pham if he could sign the consent form for treatment. The consent forms had two columns, one in English and the other in Vietnamese. The interpreter explained that what was written in Vietnamese was the same as what was written in English. The nurse brought Mr. Pham to the treatment room. Speaking through the interpreter, the nurse introduced himself as Malik Morgan and asked the names of the patient and the interpreter. When the physician entered the room, the nurse introduced the patient and interpreter. He also stated that the patient spoke Vietnamese. The physician introduced herself as Dr. Lepeche and asked the patient what had happened. The patient explained through the interpreter that he had fallen down the last three steps in front of his home and injured his wrist. He also stated that his wrist was in severe pain and that anytime he moved it, the pain became worse. The physician explained that she would order an x-ray. She then asked the patient if he was taking any medications, had any allergies to medical, or serious medical conditions. The patient stated that he had once been given codeine and had a bad reaction to it. He had been told never to take it again. The physician told the nurse that she would prescribe Toradol instead of Codeine for the pain. The PSC, nurse, and physician all must have received training on how to interact with an interpreter. Rather than speaking directly to the interpreter and ignoring the patient, they all spoke directly to the patient, while the interpreter facilitated communication. In a much-improved encounter, the patient's adverse drug reaction was avoided and the level of patient satisfaction likely improved.

\section{Ethical issues}

Not applicable.

\section{Competing interests}

The author declares that she has no competing interests.

\section{Authors' contributions}

$\mathrm{CCW}$ is the single author of the manuscript.

\section{References}

1. Breaking down the Language Barrier: Translating Limited English Proficiency into Practice (Videos available from the U.S.
Department of Justice Civil Rights Division at http://www.justice. gov/crt/pressroom/videos. php?group $=2$

2. Texas Association of Healthcare Interpreters and Translators (TAHIT). Public Service Announcement [internet]. Available from: http://www.youtube.com/watch?v=UBLuaoGXOBg

3. U.S. Census Bureau, 2010 Census.

4. U.S. Census Bureau, 2011 American Community Survey.

5. Pandya C, Batalova J, McHugh M. Limited English Proficient Individuals in the United States: Number, Share, Growth, and Linguistic Diversity. Washington, DC: Migration Policy Institute; 2001.

6. U.S. Department of Health and Human Services, Office for Civil Rights. Guidance to Federal Financial Assistance Recipients Regarding Title VI Prohibition against National Origin Discrimination Affecting Limited English Proficient Persons [internet]. Available from: http://www.hhs.gov/ocr/civilrights/resources/laws/revisedlep. html

7. U.S. Department of Health and Human Services, Office of Minority Health. Final Report: National Standards for Culturally and Linguistically Appropriate Services in Health Care, 2001.

8. U.S. Department of Health and Human Services, Office of Minority Health. National Standards for Culturally and Linguistically Appropriate Services (CLAS) in Health and Health Care [internet]. 2013. Available from: https://www.thinkculturalhealth.hhs.gov/content/clas.asp

9. Institute of Medicine. Unequal Treatment: Confronting Racial and Ethnic Disparities in Health Care. Washington, DC: National Academies Press; 2002.

10. The Joint Commission Sentinel Event Database [internet]. Available from: http://www.jointcommission.org/sentinel event.aspx

11. Flores G. The Impact of Medical Interpretation Services on the Quality of Health Care: A Systematic Review. Medical Care Res Rev 2005; 62: 255-99.

12. Flores G, Laws MB, Mayo SJ, Zuckerman B, Abreu M, Medina L, et al. Errors in Medical Interpretation and Their Potential Clinical Consequences in Pediatric Encounters. Pediatrics 2003; 111: 6-14.

13. Divi C, Koss RG, Schmaltz SP, Loeb JM. Language Proficiency and Adverse Events in U.S. Hospitals: A Pilot Study. Int J Qual Health Care 2007; 19: 60-7.

14. Weise E. Demand Surges for Medical Translators at Medical Facilities. USA Today [serial on the internet]. 2006 July 20. [cited 2013 Nov 2]; Available from: http://www.usatoday.com/news/ health/2006-07-19-medical-interpreters_x.htm

15. Diamond LC, Schenker Y, Curry L, Bradley EH, Fernandez A. Getting By: Underuse of Interpreters by Resident Physicians. $J$ Gen Intern Med 2008; 24: 256-62.

16. Ebden P, Carey OJ, Bhatt A, Harrison B. The Bilingual Consultation. Lancet 1988; 1: 347.

17. Flores G, Abreu M, Schwartz I, Hill M. The Importance of Language and Culture in Pediatric Care: Case Studies from the Latino Community. J Pediatr 2000; 137: 842-8.

18. Harsham P. A Misinterpreted Word Worth $\$ 71$ Million. Med Econ 1984; 61: 289-92.

19. Health Research \& Educational Trust. Hospital Language Services for Patients with Limited English Proficiency: Results from a National Survey [internet]. 2006. Available from: http://www.hret. org/resources/1550998119

20. Martinez EL. White Paper: For the Health Care CEO: Thinking about Language Access in Health Care [internet]. 2008. Available from: http://www.pgsi.com/Products/Resources/WhitePapers/WhitePaper1. aspx\#form 